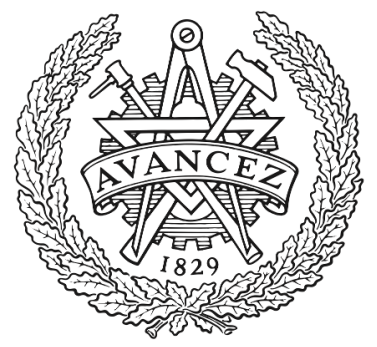

CHALMERS

UNIVERSITY OF TECHNOLOGY

\title{
Transport Properties and Local Structure of an Imidazole/Protic Ionic Liquid Mixture Confined in the Mesopores of Hydrophobic Silica
}

Downloaded from: https://research.chalmers.se, 2023-04-26 09:45 UTC

Citation for the original published paper (version of record):

Vavra, S., Elamin, K., Evenäs, L. et al (2021). Transport Properties and Local Structure of an Imidazole/Protic Ionic Liquid Mixture Confined

in the Mesopores of Hydrophobic Silica. Journal of Physical Chemistry C, 125(4): 2607-2618.

http://dx.doi.org/10.1021/acs.jpcc.0c08627

N.B. When citing this work, cite the original published paper. 


\title{
Transport Properties and Local Structure of an Imidazole/Protic lonic Liquid Mixture Confined in the Mesopores of Hydrophobic Silica
}

\author{
Szilvia Vavra, Khalid Elamin, Lars Evenäs, and Anna Martinelli* \\ Cite This: J. Phys. Chem. C 2021, 125, 2607-2618 \\ Read Online
}

ACCESS | Lلll Metrics \& More | 国 Article Recommendations ｜ sl Supporting Information

ABSTRACT: The local structure and the molecular dynamics of an imidazole/protic ionic liquid mixture have been investigated while confined in only ca. $5 \mathrm{~nm}$ mesopores of silica particles. The walls of the silica pores were functionalized with trioctyl groups to ensure a hydrophobic character, and a series of hybrid materials with varying liquid-to-silica ratios were investigated. Results from vibrational spectroscopy (both Raman and infrared) indicate that the local ion-ion interactions as well as the nature of hydrogen bonds inside the nanopores are not significantly different from the case of the bulk liquid mixture.

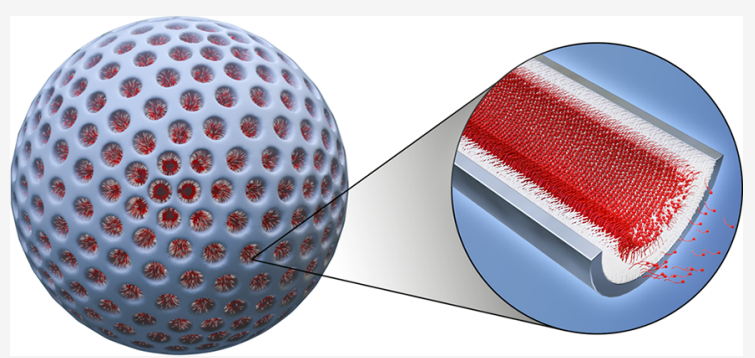
Nevertheless, the ionic conductivity decreases rapidly and monotonically with decreasing amount of liquid, while the self-diffusion coefficients measured by pulsed field gradient nuclear magnetic resonance (NMR) show a distinct dependence on composition. The population of molecules outside the particles seems to contribute with an enhanced diffusivity, while the molecules inside the mesopores diffuse at a rate comparable to that observed in the bulk liquid. In addition, when experimentally possible, we have measured higher diffusivities for the exchangeable - $\mathrm{NH}$ proton than for any other molecular species, which is indicative of a decoupled proton motion. Results from X-ray scattering, employed to elucidate the local molecular structure, reveal an additional feature characteristic of the nanoconfined state, which is associated with a real space distance of about $3.5 \mathrm{~nm}$. This distance describes a specific molecular organization inside the mesopores and may reflect the formation of a monolayer of the octyl-imidazolium cations self-assembled at the hydrophobic silica surface. Such a local structure would favor the localization of charges, including the exchangeable protons. In addition, the analysis of molar conductivity suggests that a major problem with a low pore filling is the emergence of discontinuities throughout the liquid phase.

\section{INTRODUCTION}

Proton transport mechanisms and correlated local structures of protic ionic liquids (PILs) in nanoconfinement are of increasing interest since they fundamentally determine their proton conduction properties. ${ }^{1-3}$ Proton conduction in PILs is studied not only in the perspective of a scientifically interesting charge transport phenomenon but also as a phenomenon that has potential applicability in electrochemical devices. ${ }^{1-9}$ Indeed, proton-conducting PILs are considered to be suitable electrolytes for low- and intermediate-temperature fuel cells, ideally operating above $120{ }^{\circ} \mathrm{C}$ and under anhydrous conditions, as required for the next generation of proton exchange membranes (PEMs). ${ }^{1-9}$ Since fuel cells require a solidlike electrolyte to operate, a common approach is to confine PILs in the pores of a solid matrix, thus forming a functional hybrid material. The aim is to circumvent the practical problem of leakage that is intrinsically associated with the liquid state of matter. ${ }^{1-4,7,8,10}$ Hence, investigating the effect of confinement on the desired physical and chemical properties of PILs is essential, especially if the confinement occurs on the nanoscale. ${ }^{1-4,8}$ Indeed, the anomalous transport behavior of molecular liquids observed in nanoconfinement is a subject of relevant and progressive contemporary research. ${ }^{11-13}$ In the same line, computational and experimental effort has been made to elucidate the local structure of ILs confined in nanodomains of, e.g., silica or graphite. ${ }^{14-17}$ One should note, however, that experimental works on nanoconfined ILs are more limited in number compared to the computational ones.

PILs, differently from conventionally known ionic liquids, are distinct by possessing an exchangeable proton, most commonly on the cation, that has the potential to contribute to the overall proton conduction via the Grotthuss mechanism (i.e., by proton hopping), in addition to the vehicular type of charge transport that is typical of liquid electrolytes. ${ }^{5,6,9,18}$ During the vehicular type of charge transport, the proton is chemically bound to the cation and the ionic conductivity is

Received: September 22, 2020

Revised: January 12, 2021

Published: January 27, 2021 
limited by the diffusion rate of the molecular ionic species. ${ }^{5}$ However, provided an appropriate hydrogen-bonded network, protons could move by the Grotthuss mechanism much faster than the diffusing parent molecules, by means of a dynamic reorganization of the involved hydrogen bonds. ${ }^{5,6,9}$ In the case of alkyl-imidazolium-based PILs, the imidazolium head of the cation can provide proton donor sites, while proton acceptor sites can strategically be included by, for instance, the addition of an amphoteric molecule like imidazole; ${ }^{1,6,19}$ albeit triazole and pyrazole should hypothetically have a similar effect.

In the context of electrochemical devices that operate by proton conduction, there is an obvious interest in materials that can provide the conditions for a thermodynamically favored Grotthuss mechanism. In the specific case of PILs, we know from experimental and computational studies that a too high ionicity (typically a $\left.\Delta \mathrm{p} K_{\mathrm{a}}>17\right)^{5}$ decreases the proton mobility due to extremely (chemically) stable ionic species. On the other hand, much lower $\Delta \mathrm{p} K_{\mathrm{a}}$ values are associated with stable neutral parent molecules, which results in molecular mixtures rather than truly ionic systems. ${ }^{5,6,9}$ Thus, the specific choice of the ionic pair is crucial in determining the local interactions and hence the mechanism of charge transport in PILs; possibilities here are vast since ionic liquids are also known as "designer solvents" because of the immense number of theoretically possible cation-anion pairs.

Nanoconfinement of a PIL can influence the diffusivity of the constituting ions (both anions and cations) as well as the nature of the established hydrogen bonds, resulting in changed proton conduction properties compared to the bulk liquid state. ${ }^{1,2}$ Within the framework of organic-inorganic hybrid materials made of imidazolium ionic liquids and mesoporous silica, the effect of nanoconfinement has been primarily studied for the case of aprotic ionic liquids, ${ }^{2,4,8,20-23}$ with fewer contributions focused on the protic analogs. ${ }^{1-3}$ Aprotic and protic ionic liquids based on the alkyl-imidazolium cation have in common the nanosegregation into polar and nonpolar domains even in their liquid state, provided an alkyl chain on the cation longer than butyl that increases the amphiphilic nature of the cation. ${ }^{24,25}$ Likewise, if confined in pores of silica, both aprotic ${ }^{20,21}$ and protic $^{1,2}$ alkyl-imidazolium ionic liquids show that their local structure on the nanoscale is strongly influenced by the chemistry of the pore walls.

If the silica walls are chemically untreated, thus exposing silanol groups $(-\mathrm{SiOH})$ and nonbridging oxygens $\left(-\mathrm{SiO}^{-}\right)$, the polar domains of the ionic liquid orient toward the pore walls due to anion-silica and cation-silica interactions, leading to a dramatic decrease of the diffusivity of mainly the imidazolium cation, measurable by pulsed field gradient (PFG) nuclear magnetic resonance (NMR), and of the macroscopically observed ionic conductivity, typically measured by impedance spectroscopy. ${ }^{1,2,20,21}$ By contrast, hydrophobization of the silica walls leads to the so-called flipped-ion effect, by which the nonpolar domains of the cation (i.e., alkyl chains) assemble at the pore wall. With this molecular orientation, there are only weak dispersion forces between the alkyl chains of the imidazolium cations and the hydrophobic groups anchored on the silica walls allowing unrestricted, high diffusivities. ${ }^{1,2,20,21}$ This phenomenon has been reported by Iacob et al. ${ }^{21}$ for the case of the aprotic ionic liquid 1-methyl-3butylimidazolium tetrafluoroborate that displays cation diffusivity and ionic conductivity higher inside hydrophobized mesoporous silica than in both untreated silica and the bulk liquid. On the same line, Garaga et al. ${ }^{1}$ have recently reported that the imidazole/ $\mathrm{HC}_{8}$ ImTFSI mixture confined in hydrophobized mesoporous silica displays a higher cation diffusivity than in the bulk pure ionic liquid, and also that a decoupled proton motion could occur. The latter is what distinguishes the vehicular from the Grotthuss mechanism.

In this study, we use the same imidazole/protic ionic liquid $\left(\mathrm{HC}_{8} \mathrm{ImTFSI}\right)$ mixture as the one previously presented by Garaga et al., i.e., imidazole added to $\mathrm{HC}_{8}$ ImTFSI at a molar fraction of 0.15 due to optimal proton dynamics at this composition. ${ }^{1}$ Hence, the fact that this ionic liquid has an exchangeable proton through the $-\mathrm{NH}$ group on the octylimidazolium cation and that it tends to segregate into polar and nonpolar domains in the nanometer scale has already been proven (by NMR- and X-ray-based methods) and discussed. ${ }^{1}$ More specifically, in that ${ }^{1}$ and in a previous work, ${ }^{19}$ we demonstrated that the addition of imidazole to an imidazolium-based PIL has the positive effect of mobilizing the exchangeable protons, which can thus achieve a diffusivity a few times higher than that of the cationic species. Based on this finding, the focus of the current work is now to investigate whether downsizing the pores of the hydrophobized mesoporous silica from $10 \mathrm{~nm}$ to only about $5 \mathrm{~nm}$, hence approaching the size of the confined molecular species, has an impact on the liquid uptake as well as on the molecular dynamics and/or the nanosegregated structure. In addition, we also make a composition study, where the amount of the liquid phase added to the solid phase of mesoporous silica is a variable parameter. This study provides a comprehensive investigation, which includes several complementary methods such as optical imaging, nitrogen adsorption, impedance spectroscopy, diffusion NMR, vibrational spectroscopy, and X-ray scattering, which have been selected to achieve both structural and dynamical information.

\section{EXPERIMENTAL SECTION}

Materials. The protic ionic liquid (PIL) 1-octylimidazolium bis(trifluromethylsulfonyl)imide (97\%, here abbreviated as $\mathrm{HC}_{8}$ ImTFSI), was custom-made by IoLiTec (Ionic Liquids Technologies $\mathrm{GmbH}$ ), while imidazole (97\%, henceforth abbreviated as Im) was purchased from Merck KGaA. The molecular structure of these compounds is given in Figure 1.

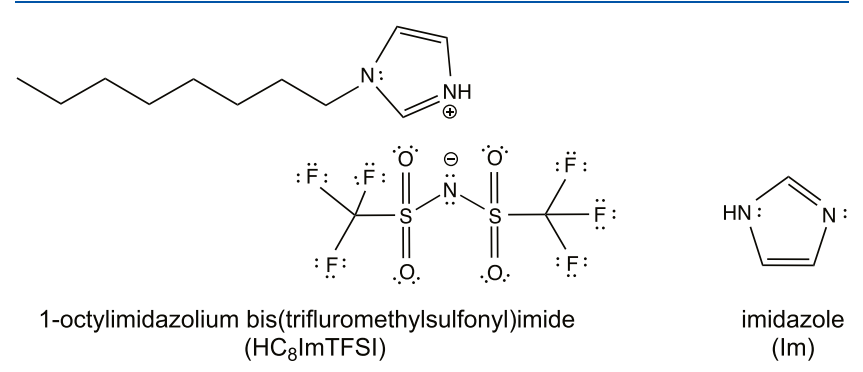

Figure 1. Molecular structure of the protic ionic liquid 1octylimidazolium bis(trifluromethylsulfonyl)imide ( $\mathrm{HC}_{8}$ ImTFSI) and imidazole (Im).

The mesoporous silica microparticles (abbreviated as 5-C8$\mathrm{SiO}_{2}$ ) were provided by Nouryon $\mathrm{AB}$ (previous Akzo Nobel Pulp and Performance Chemicals $\mathrm{AB}$ ) and synthesized with the emulsion and solvent evaporation (ESE) method. The pore walls available in these particles were functionalized with trioctylsilyl (TOS) groups. The nominal mean diameter of the 
silica particles was given to be $6 \mu \mathrm{m}$, while the average pore diameter was measured by us to be on average $5-6 \mathrm{~nm}$.

Sample Preparation. First, a liquid mixture, henceforth denoted Im/PIL, was prepared from imidazole and the PIL, $\mathrm{HC}_{8}$ ImTFSI, in which the molar fraction $(\chi)$ of imidazole was set equal to 0.15 . Afterward, a sample series was obtained by multistep centrifugation of the Im/PIL liquid mixture and the mesoporous silica particles $\left(5-\mathrm{C} 8-\mathrm{SiO}_{2}\right)$ added at different ratios to achieve various degrees of filling of the silica pores. The centrifugation was carried out with a Labofuge 200 Heraeus Sepatech centrifuge and further details on the centrifuging procedure can be found in the Supporting Information (Tables S1 and S2). The nominal pore filling ranged from 50 to $400 \%$, where $100 \%$ pore filling indicates that the volume of the Im/PIL mixture added to the microparticles was calculated to just uptake the entire free volume of the available mesopores. Similarly, a pore filling lower than $100 \%$ implies partially filled pores and a pore filling larger than $100 \%$ results in some liquid occupying the space in between the microparticles. The nominal composition and other details of the investigated hybrid materials are summarized in Table 1, while their illustration is given in Figure 2.

Table 1. Composition of the Samples Obtained by Filling the Pores in 5-C8-SiO 2 Microparticles with the Im/PIL Liquid Mixture $^{a}$

\begin{tabular}{lll} 
pore filling & $\varphi_{\text {silica }}$ & \multicolumn{1}{c}{ phase } \\
$0 \%$ & 1.000 & dry powder \\
$50 \%$ & 0.560 & wet powder \\
$100 \%$ & 0.389 & wet powder \\
$200 \%$ & 0.242 & suspension \\
$400 \%$ & 0.137 & suspension \\
Im/PIL & 0.000 & liquid
\end{tabular}

${ }^{a} \varphi_{\text {silica }}$ is the resulting volume fraction of silica in the hybrid sample.

Scanning Electron Microscopy. Scanning electron microscopy (SEM) micrographs were obtained with a JEOL $7800 \mathrm{~F}$ Prime microscope using a $1.5 \mathrm{kV}$ acceleration voltage. For imaging, the secondary electrons were collected. Before imaging, the samples were coated with palladium using an EMITECH K550x sputter coater.

Nitrogen Adsorption. Nitrogen adsorption on mesoporous silica microparticles was measured with a TriStar 3000 instrument from Micromeritics after an outgassing treatment of the sample overnight at $120^{\circ} \mathrm{C}$ under a nitrogen flow. The surface area was determined using the Brunauer-EmmettTeller (BET) method on the linear range of the isotherms, typically at $p / p_{0}=0.05-0.25$. The pore size distribution of the mesopores was calculated with the Barrett-Joyner-Halenda $(\mathrm{BJH})$ method using the adsorption isotherm, while the total pore volume was determined by applying the Gurvich rule at $p / p_{0}=0.98 .^{26}$

Impedance Spectroscopy. The conductivity measurements were carried out on a Broadband Dielectric Spectrometer (Novocontrol $\mathrm{GmbH}$ ). The samples were placed between two gold-plated brass electrodes with a diameter of $13.5 \mathrm{~mm}$. The thickness of each sample was controlled to be 1 $\mathrm{mm}$ using a silica spacer. The measurements covered the $10^{-1}-10^{7} \mathrm{~Hz}$ frequency range, whereas the temperature was scanned between -105 and $155{ }^{\circ} \mathrm{C}$. The temperature was controlled using a nitrogen gas cryostat with a stability of \pm 0.5 ${ }^{\circ} \mathrm{C}$. Conductivity data were collected at intervals of $20{ }^{\circ} \mathrm{C}$ with a stabilization time of $600 \mathrm{~s}$ at each temperature.

Diffusion NMR. The diffusion NMR experiments were carried out at $30{ }^{\circ} \mathrm{C}$ and set up based on the experience from our previous work. ${ }^{1}$ A Bruker Avance 600 spectrometer was used, equipped with a Bruker Diff30 probe with a $5 \mathrm{~mm} 1 \mathrm{H} /$ $2 \mathrm{H}$ RF double-coil insert and connected to a $60 \mathrm{~A}$ gradient amplifier. The relaxation times were obtained by standard inversion recovery $\left(T_{1}\right)$ and CPMG $\left(T_{2}\right)$ pulse sequences. All NMR signals, in both liquid samples and hybrid materials (i.e., the liquid mixture inside the mesopores of silica), revealed a monoexponential behavior for the relaxation recovery. For the case of liquid samples, the standard stimulated echo sequence was used with a gradient pulse length, $\delta$, set to $1 \mathrm{~ms}$ and a diffusion time, $\Delta$, set to $100 \mathrm{~ms}$. In each experiment, the gradient strength, $g$, was ramped uniformly in 16 steps from 0.1 to $12 \mathrm{~T} / \mathrm{m}$. Data were collected at each gradient value with a sum of 8 acquisitions, and using a $7 \mathrm{~s}$ recycle delay time to ensure complete longitudinal relaxation of all signals. For the hybrid samples, the stimulated echo sequence using bipolar pulses was used to suppress the influence of internal background gradients. Due to fast spin-spin relaxation, a gradient pulse length $\delta$ equal to $0.37 \mathrm{~ms}$ was used, while the diffusion time $\Delta$ was set to $300 \mathrm{~ms}$. In each experiment, the gradient strength $g$ was ramped uniformly in 16 steps from 0.1 to $17 \mathrm{~T} / \mathrm{m}$. Data were collected at each gradient value with a sum of eight acquisitions, and using a $4 \mathrm{~s}$ recycle delay time to ensure complete longitudinal relaxation of all signals. The gradient strength was calibrated with the self-diffusion coefficient of HDO traces in a standard sample of pure $\mathrm{D}_{2} \mathrm{O}$. For all samples, a one-component attenuation of the logarithmic NMR signal intensity against $k$ was noted, where $k$ is equal to $(\gamma g \delta)^{2} \cdot(\Delta-\delta / 3)$ and $\gamma$ is the ${ }^{1} \mathrm{H}$ gyromagnetic ratio.

X-ray Scattering. Small- and wide-angle X-ray scattering (SAXS and WAXS) measurements were performed on a Mat:Nordic instrument from SAXSLAB/Xenocs. The X-ray beam was produced by a $\mathrm{Cu}$-radiation source and focused with a Micro-Max 003 X-ray generator from Rigaku. A Pilatus 300K from Dectris was used as the detector. The samples were kept

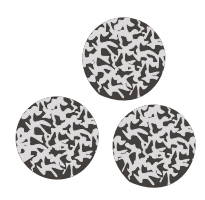

$0 \%$

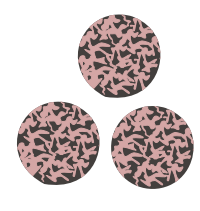

$50 \%$

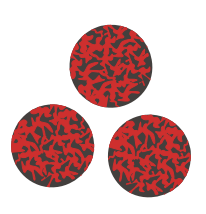

$100 \%$

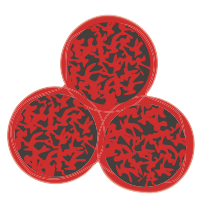

$200 \%$

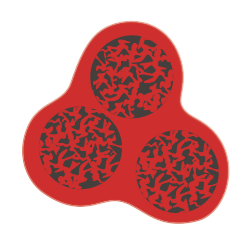

$400 \%$

Figure 2. Illustration of, from left to right, the bare mesoporous silica microparticles and the same particles with the mesopores filled by an increasing amount of the liquid mixture. 
inside quartz capillaries with an inner diameter of $0.15 \mathrm{~mm}$ (Hilgenberg). For calibration, silver behenate was used. The sample-to-detector distance was $134 \mathrm{~mm}$, and the exposure time was $480 \mathrm{~s}$. The collected two-dimensional (2D) scattering patterns were further processed with SAXSGUI (Rigaku) software to transform them into one-dimensional (1D) scattering graphs. The size of scattering objects was estimated from an observed structural correlation length, $d$, where $d=$ $2 \pi / q_{\max }$ and $q_{\max }$ is the position of the scattering peak. To obtain these dimensions, the experimentally collected X-ray scattering patterns were fitted with a multicomponent function that includes a linear contribution (for the background) and a number of Gaussian components (see an example in Figure S1).

Vibrational Spectroscopy. The infrared (IR) spectra were recorded on a PerkinElmer Fourier transform infrared (FT-IR) spectrophotometer in the attenuated total reflectance (ATR) mode using a single-reflection diamond ATR crystal (GladiATR, Pike Technologies). The full optical range of 4000 to $400 \mathrm{~cm}^{-1}$ was covered, with a spectral resolution of $1 \mathrm{~cm}^{-1}$. However, since a diamond ATR crystal was used, the spectra were cut between 1900 and $2600 \mathrm{~cm}^{-1}$. For all experiments, 64 scans were collected and averaged. The recorded data were subjected to baseline correction. To correctly interpret infrared intensities, as well as their spatial origin, we have calculated the penetration depth, $d_{\mathrm{p}}$, of the infrared radiation as a function of wavenumber (see Figure $S 2$ ). From the character of $d_{\mathrm{p}}$ and the analysis of selected regions of the infrared spectra, we conclude that also the bulk of the particles contribute to the recorded spectral intensities (Figures S3-S5).

Raman spectra were recorded with an InVia Reflex Raman spectrophotometer from Renishaw equipped with a chargecoupled device (CCD) detector. A $532 \mathrm{~nm}$ wavelength diode laser was used as the incident light while the grating used had 2400 grooves $/ \mathrm{mm}$. During the $2 \mathrm{D}$ mapping measurements, the confocal mode and a $\times 100$ long working distance (LWD) Leica objective with a numerical aperture (NA) of 0.75 were used. With this experimental setup, a spectral resolution of 1 $\mathrm{cm}^{-1}$ and a spatial resolution of about $0.8 \mu \mathrm{m}$ (approximating to the relation $1.22 \cdot \lambda / \mathrm{NA}$, where $\lambda$ is the wavelength of the incoming laser) could be achieved. During the mapping measurements, the laser power was set to around $20 \mathrm{~mW}$, while the acquisition time was adapted to the sample investigated but was typically kept between 10 and $20 \mathrm{~s}$. Point spectra within the particles were taken with a $200 \mathrm{~mW}$ laser power by averaging 10 scans with an acquisition time of 10 s. The collected Raman spectra did not suffer from major problems of fluorescence and hence of a strong interfering background. For example, in the narrow spectral range 720$760 \mathrm{~cm}^{-1}$ used to extract integrated areas, a simple fit based on a linear background and a Lorentzian function was used. Before each measurement, the spectrophotometer was calibrated to the first-order vibrational mode of a $\mathrm{Si}$ wafer centered at $520.6 \mathrm{~cm}^{-1}$.

\section{RESULTS AND DISCUSSION}

Morphology of the Mesopores. The effect of nanoconfinement on the local structure and dynamics of the Im/ PIL mixture was studied in hydrophobized silica mesopores. To enable an in-depth study, the morphology of the pores was first characterized. Since the mesoporous silica was prepared with the ESE method, the silica microparticles were spherical in shape and highly monodisperse in size, as can be seen in the inset of Figure 3B.
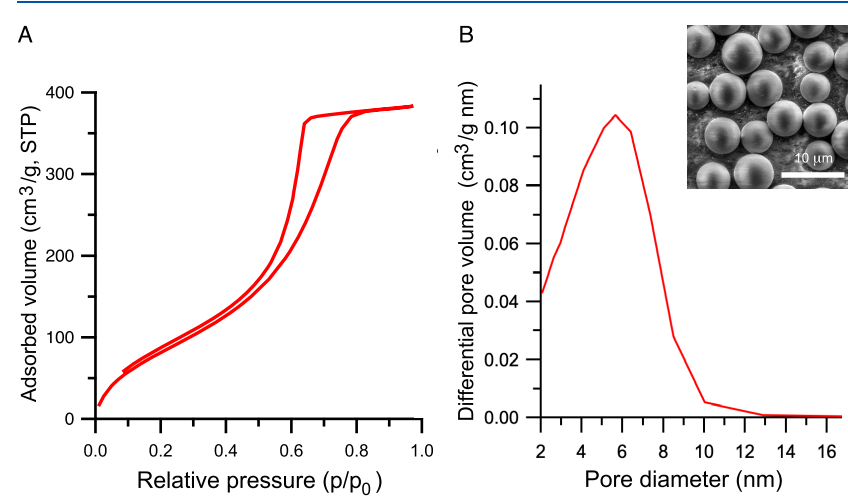

Figure 3. Nitrogen adsorption-desorption isotherms at $77 \mathrm{~K}$ (A), and differential pore size distribution (B), obtained for the neat 5-C8$\mathrm{SiO}_{2}$ microparticles. The top-right inset shows a scanning electron microscopic (SEM) image of the pristine $5-\mathrm{C} 8-\mathrm{SiO}_{2}$ mesoporous silica microparticles.

The pores within these microparticles showed a nitrogen adsorption curve typical of mesoporous materials with adsorption-desorption isotherms exhibiting a clear H2-type hysteresis loop (Figure 3A). Results from the BrunauerEmmett-Teller (BET) analysis revealed that the available free volume in the $5-\mathrm{C} 8-\mathrm{SiO}_{2}$ microparticles is $0.59 \mathrm{~cm}^{3} / \mathrm{g}$ and the specific surface area is $352 \mathrm{~m}^{2} / \mathrm{g}$. Moreover, the $\mathrm{C}$-value of 5 $\mathrm{C} 8-\mathrm{SiO}_{2}$ (the $\mathrm{C}$-value is also known as the energetic parameter and is obtained from BET fitting ${ }^{27}$ ) was very close to the $C$ value of similar TOS-functionalized particles previously investigated by our group ${ }^{1}$ (see data in Table S3). Those microparticles were prepared with the same procedure, but had a larger pore diameter of $10 \mathrm{~nm}$. Such similar $C$-values indicate that their isosteric enthalpies of nitrogen adsorption are very close; hence, it can be assumed that they have the same surface density of hydrophobic TOS groups. In addition, it can also be observed that the $C$-value is significantly higher for nonfunctionalized mesoporous silica microparticles, revealing that hydrophobization with TOS groups leads to a decreased isosteric enthalpy of nitrogen adsorption.

Regarding the local morphology, the pore size distribution in 5- $\mathrm{C} 8-\mathrm{SiO}_{2}$ was found to be relatively narrow with a maximum at $5.6 \mathrm{~nm}$ (Figure $3 \mathrm{~B}$ ). Also, since these pores result from a disordered bicontinuous silica structure, they do not contribute with sharp features in the X-ray scattering pattern (see also Figure 11 and the related discussion below).

Truly Filled Mesopores. Chemical mapping performed with a Raman spectrometer in the confocal mode proved that for every degree of pore filling the Im/PIL mixture penetrated the bulk of the 5-C8-SiO ${ }_{2}$ microparticles and distributed evenly throughout the mesopores. Confocal Raman spectroscopy is an excellent method for chemical mapping in, e.g., $2 \mathrm{D}$, and has been applied here for single layers of microparticles to verify the spatial location of the liquid phase (not, however, as a method to determine the pore filling factor). Figure 4 shows a schematic of the working principles for the representative case of the $100 \%$ filled particles. In brief, over a selected area of proximate particles (a) a 2D mapping experiment is performed keeping the confocal point, hence the volume from where the Raman intensity is collected, fixed at a half-diameter height, i.e., at $d / 2$, which here is equal to approximately $3 \mu \mathrm{m}$ from the 


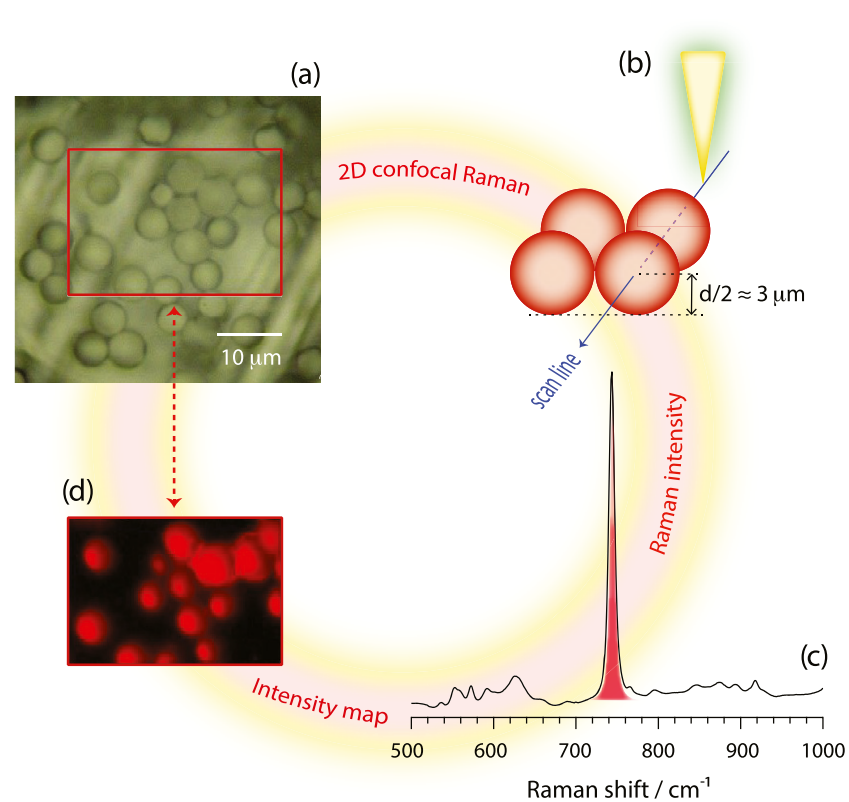

Figure 4. (a) Microscope image of a monolayer of silica microparticles filled by $100 \%$ with the $\mathrm{Im} / \mathrm{PIL}$ liquid mixture. (b) Geometrical details of the 2D Raman mapping in confocal mode with focus positioned at half-height $(d / 2)$ with respect to the particles' diameter. (c) Raman intensity used as a probe for the location in space of the liquid mixture, i.e., the peak at $742 \mathrm{~cm}^{-1}$ characteristic of the TFSI anion. (d) Result of the intensity analysis over space, showing a perfect match with the shape and size of the particles shown in (a) and indicating the presence of the liquid in the entire spherical volume.

bottom of the particles (b). The most intense Raman peak, that here is the feature at $\sim 742 \mathrm{~cm}^{-1}$ arising from the expansion-contraction mode of the TFSI anion, is used as the probe signal (c) to obtain spatial information on the liquid phase (d). The 2D map shown in Figure $4 d$ is created by transforming the integrated intensity underlying the peak at $742 \mathrm{~cm}^{-1}$ into a color code at every collection point (red for more intense and black for less intense; please note that black does not necessarily mean null intensity). This shows to perfectly reproduce the shape and dimension of the single particles investigated and previously imaged by a built-in camera of the microscope (a). The chemical maps obtained in the same way from samples with different filling ratios are summarized in Table S4. The obtained 2D chemical maps confirm that the liquid phase has penetrated the entire volume of the particles and hence is distributed within the mesopores. Had the liquid phase not penetrated the pores available in the bulk of the particles, a peripheral intensity map would have been recorded instead, which is clearly not the case here.

A very intriguing observation is that for the samples with filling factors of 200 and $400 \%$, the intensity of the Ramanactive mode at $\sim 742 \mathrm{~cm}^{-1}$ appeared higher for focal points inside the microparticles and lower for focal points outside the microparticles, as summarized in Figure 5. Although being outside the scope of the current work, this phenomenon is worth being discussed. The observed intensity enhancement cannot be explained by a local concentration difference, since the volume occupied by the silica walls should rationally result in the opposite contrast, that is lower intensities for the TFSI feature inside the particles than in the bulk liquid residing outside the particles. Moreover, higher intensities were also
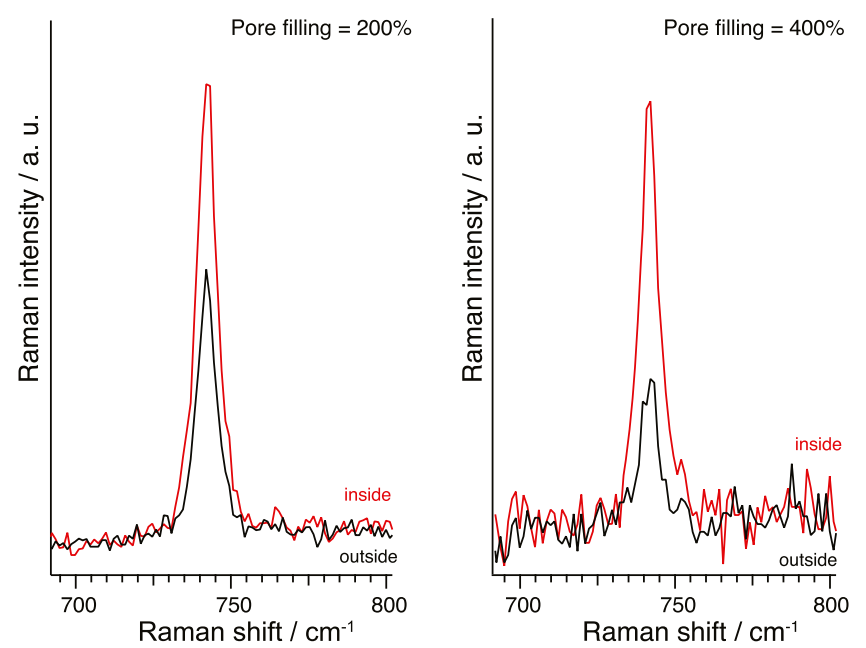

Figure 5. Raman spectra in the region of the expansion-contraction mode of the TFSI anion, extracted from 2D chemical mapping at points inside and outside the microparticles.

detected for the Raman feature at around $2900 \mathrm{~cm}^{-1}$ (i.e., the $\nu_{\mathrm{s}}$ and $\nu_{\mathrm{as}} \mathrm{C}-\mathrm{H}$ modes of the alkyl chains) indicating that the enhancement is not a consequence of an inhomogeneous distribution of the ionic species. Based on these argumentations, we raise the hypothesis that the detected intensity enhancement is due to multiple reflections of light inside the nanoporous structure of the silica microparticles. This is possibly a new material concept able to enhance the intensity of Raman scattered light, with an underlying mechanism closely related to the concepts of nonplasmonic surfaceenhanced Raman scattering using silica microspheres ${ }^{28}$ and of light trapping within nanoholes in silicon. ${ }^{29}$ These very recent works emphasize new means to enhance the Raman signal, which are different from the already established phenomena of plasmonic resonance ${ }^{30}$ and whispering-gallery mode resonance. ${ }^{31}$

Ionic Conductivity. The effect of nanoconfinement on the dynamics of the Im/PIL liquid phase was investigated by impedance spectroscopy, through the analysis of ionic conductivity. The measured ionic conductivities are shown in Figure 6 as a function of temperature, between -105 and 155 ${ }^{\circ} \mathrm{C}$, in an Arrhenius plot. For all samples, the conductivity shows a nonlinear dependence on inverse temperature, which is the typical case of ionic liquids and liquid electrolytes when the transport of charges is dominated by the vehicular mechanism. In addition, we observe no abrupt changes in conductivity within this temperature range, indicating that no phase transitions, i.e., solid-to-liquid, occur in the investigated temperature window. The pure ionic liquid $\mathrm{HC}_{8}$ ImTFSI does not display any phase transitions either, as discussed in the recent work of Abdurrokhman et al., ${ }^{25}$ but behaves as a glassforming liquid with a $T_{\mathrm{g}}$ at $-80{ }^{\circ} \mathrm{C}$. Also, both confinement inside mesopores of silica ${ }^{22,32,33}$ and addition of imidazole to an imidazolium ionic liquid ${ }^{19}$ typically favor a disordered liquid structure and frustrate crystallization, whereby the observation above is not unexpected.

We have fitted the experimental conductivity data with the empirical Vogel-Fulcher-Tammann (VFT) equation $\sigma_{\mathrm{VFT}}=$ $\sigma_{\infty} \cdot \mathrm{e}^{-\left(D T_{0}\right) /\left(T-T_{0}\right)}$, as proposed in many other previous works and well summarized in the work of Sippel et al. ${ }^{18}$ In the VFT equation, $\sigma_{\infty}$ is the conductivity extrapolated for infinite temperatures, $D$ is a parameter related to fragility, and $T_{0}$ is a 


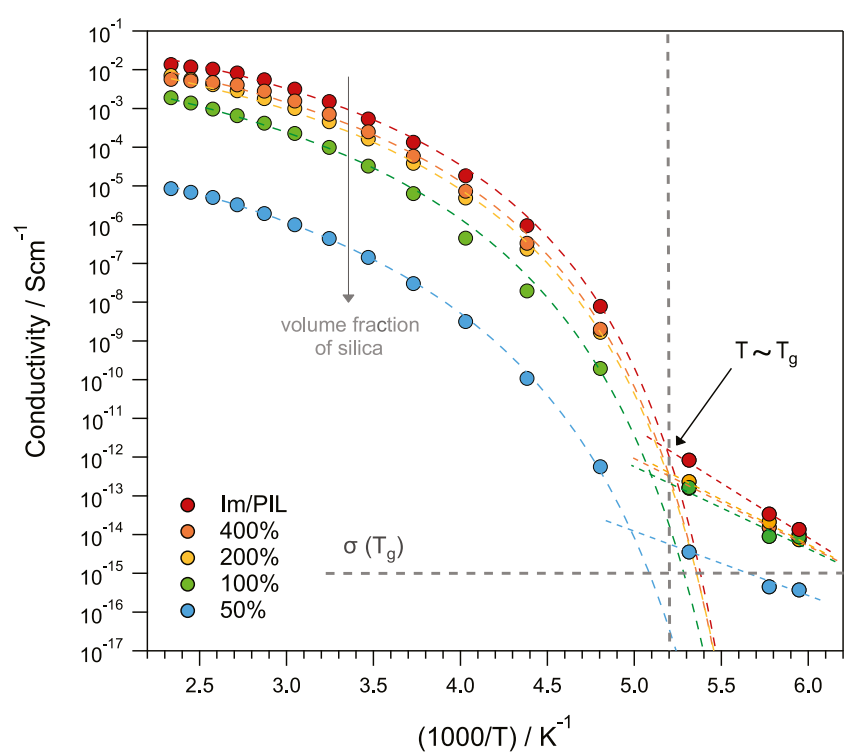

Figure 6. Arrhenius plot of conductivity values measured for the different samples investigated and covering the temperature range of -105 to $155{ }^{\circ} \mathrm{C}$. The horizontal dashed line marks the ionic conductivity expected at $T_{\mathrm{g}}\left(\sigma\left(T_{\mathrm{g}}\right) \sim 10^{-15} \mathrm{~S} / \mathrm{cm}\right)$ if the VogelFulcher-Tammann (VFT) behavior was valid over the whole $T$ range. Instead, values 2 or 3 orders of magnitude higher are measured and a linear behavior is observed at temperatures below $T_{\mathrm{g}}$.

temperature related to, and typically lower than, the glasstransition temperature $T_{\mathrm{g}}$. From the best fits results, we observe that $D$ changes only slightly with composition, and takes values between 5 and 7 (Table 2). These are all in the

Table 2. Values of $D, T_{0}$, and $\sigma_{\infty}$, as Found from Fitting the Conductivity Data with the Empirical VFT Equation $\sigma_{\mathrm{VFT}}=$ $\sigma_{\infty} \cdot \mathrm{e}^{-\left(D T_{0}\right) /\left(T-T_{0}\right)}$

\begin{tabular}{llll} 
pore filling & $\sigma_{\infty}(\mathrm{S} / \mathrm{cm})$ & $D$ & $T_{0}(\mathrm{~K})$ \\
$0 \%$ & & & \\
$50 \%$ & 0.0005 & 6.8 & 157 \\
$100 \%$ & 0.07 & 6.3 & 158 \\
$200 \%$ & 0.17 & 5.7 & 159 \\
$400 \%$ & 0.23 & 5.5 & 160 \\
$\mathrm{Im} / \mathrm{PIL}$ & 0.42 & 5.2 & 161 \\
\hline
\end{tabular}

range that classifies fragile liquids. The value of $T_{0}$ is very little dependent on the degree of pore filling, while $\sigma_{\infty}$ clearly decreases with decreasing relative amount of the liquid phase, which is observed for any temperature (as a guide, see the direction of the vertical arrow in Figure 6).

The decreased conductivity upon increased volume fraction of silica (or lower degree of pore filling) is better illustrated in Figure 7, for the arbitrarily selected temperatures of 15 and 95 ${ }^{\circ} \mathrm{C}$. This decrease, which is significant even if compensating for the volume fraction of silica, may be attributed to changed or lost connectivity between the ionic species, since the solid phase of porous silica creates longer and likely tortuous ion diffusion pathways as well as discontinuities in the longer spatial range. On this line, it is reasonable that the samples described as wet powder (50 and $100 \%$ filling) and suspension (200 and $400 \%$ filling) display pairwise similar behaviors. Finally, it is relevant to mention that the 200 and $400 \%$ samples show an ionic conductivity of $\sim 5 \times 10^{-3} \mathrm{~S} / \mathrm{cm}$, at a temperature of $120{ }^{\circ} \mathrm{C}$ and under the flow of dry $\mathrm{N}_{2}$. This

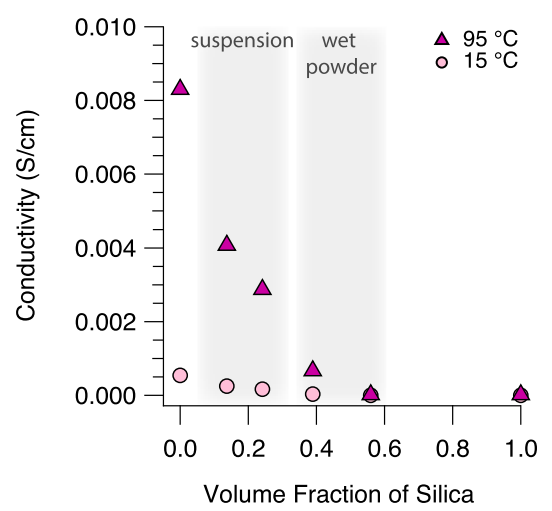

Figure 7. Absolute values of conductivity measured at 15 and $95{ }^{\circ} \mathrm{C}$ as a function of the volume fraction of silica.

value is comparable to the ionic conductivity typically observed with polymer electrolytes based on ionic liquids (between $10^{-4}$ and $10^{-3} \mathrm{~S} / \mathrm{cm}$ at room temperature), ${ }^{34-38}$ similar to or higher than the proton conductivity showed by archetypical proton conductors such as imidazole or benzimidazole at $120^{\circ} \mathrm{C}$ and anhydrous conditions $\left(2.2 \times 10^{-5}\right.$ and $1.3 \times 10^{-3} \mathrm{~S} / \mathrm{cm}$, respectively), ${ }^{39}$ and higher than the proton conductivity of perfluorinated polymers that easily dehydrate and hence perform exclusively at temperatures below $80{ }^{\circ} \mathrm{C}$ and relative humidities above $35 \%$ (e.g., at a low degree of hydration the proton conductivity of Nafion is well below $10^{-4} \mathrm{~S} / \mathrm{cm}$ and rapidly decays further with a decreasing number of hydrating water molecules). ${ }^{40}$ Another interesting observation is the transition from a VFT to an Arrhenius behavior at 1000/T values close to 5.2 (see the vertical dashed line in Figure 6). Such a transition is typically observed when a liquid is cooled down to the glassy state; ${ }^{41}$ indeed the $1000 / T$ value of 5.2 is close to $-80{ }^{\circ} \mathrm{C}$ and coincides with the $T_{\mathrm{g}}$ measured by differential scanning calorimetry (DSC) for $\mathrm{HC}_{8} \mathrm{ImTFSI}$, as mentioned above.

Self-Diffusion. The transport properties of the Im/PIL mixture while confined in the mesopores of $5-\mathrm{C} 8-\mathrm{SiO}_{2}$ microparticles were also monitored by diffusion PFG NMR measurements. The self-diffusion coefficients were calculated using the following ${ }^{1} \mathrm{H}$ resonances: the resonance at 9-11 ppm attributed to the $-\mathrm{NH}$ group (to which both imidazole and imidazolium contribute), the resonances at 6-8 ppm attributed to the $-\mathrm{C}^{2} \mathrm{H}$ and $-\mathrm{C}^{4,5} \mathrm{H}$ groups of the octylimidazolium head, as well as the resonance below $2 \mathrm{ppm}$ attributed exclusively to the $\mathrm{CH}_{2}$ and $\mathrm{CH}_{3}$ groups of the octyl chain in the octyl-imidazolium cation, Figure 8. The latter resonance is particularly important since, by not being involved in proton exchange phenomena, it provides the reference selfdiffusion coefficient of the imidazolium cation, $D_{\text {cat }}$. The selfdiffusion coefficients obtained in this study for the hybrid samples prepared with $5-\mathrm{C} 8-\mathrm{SiO}_{2}$ are given in Table 3, which also includes the values obtained from the neat $\mathrm{Im} / \mathrm{PIL}$ mixture.

The determination of self-diffusion coefficients was, however, not possible for all samples and resonances since significant broadening appears for the lowest values of pore filling. Line broadening in liquid state NMR can reflect a slowdown in molecular dynamics, but has here also contributions from the intrinsic heterogeneity of the hybrid samples. ${ }^{42,43}$ An additional aspect related to line broadening is that the resonances attributed to imidazole are not resolved 


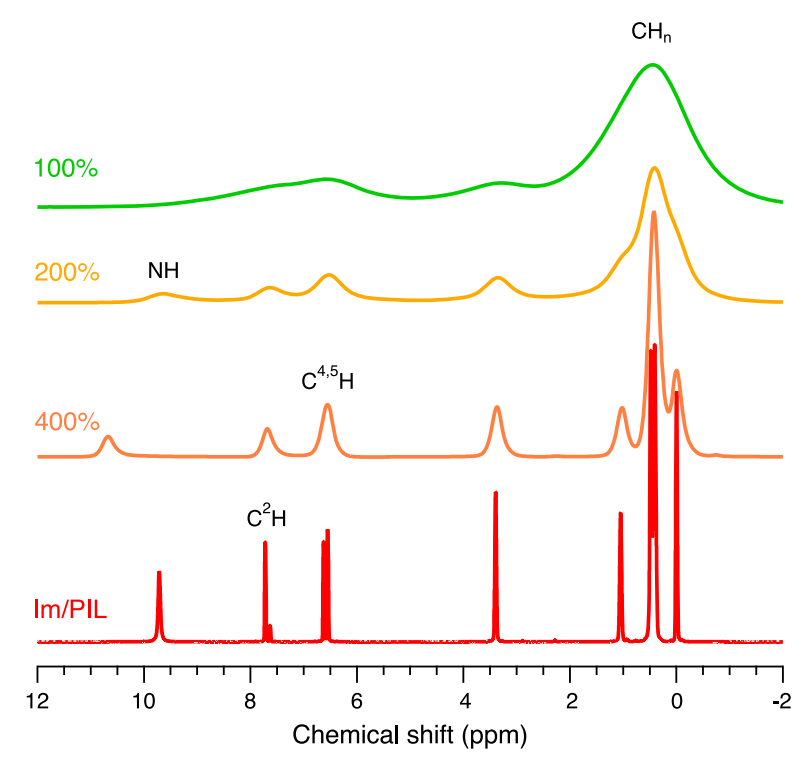

Figure 8. Selection of ${ }^{1} \mathrm{H}$ NMR spectra recorded for the investigated samples. Characteristic resonances of the imidazolium cation are labeled.

Table 3. Self-Diffusion Coefficients (in Units of $10^{-11} \mathrm{~m}^{2} / \mathrm{s}$ ) Measured for the Different Samples Using Selected ${ }^{1} \mathrm{H}$ Resonances

$\begin{array}{llllll}\text { pore filling } & D_{\text {cat }} & D_{\mathrm{NH}} & D_{\text {Im }} & D_{\mathrm{H}} & D_{\mathrm{H}} / D_{\text {cat }} \\ 0 \% & & & & & \\ 50 \% & 0.95 & \text { n.a. } & \text { n.a. } & & \\ 100 \% & 0.87 & \text { n.a. } & \text { n.a. } & & \\ 200 \% & 1.63 & 2.31 & 2.12^{b} & 5.5^{b} & 3.4^{b} \\ 400 \% & 1.66 & 2.60 & 2.38^{b} & 7.2^{b} & 4.3^{b} \\ \operatorname{Im} / \text { PIL }^{a} & 0.86 & 1.35 & 1.24 & 3.7 & 4.3\end{array}$

${ }^{a}$ Data retrieved from a previous work (see ref 1.$)^{b}$ Values estimated under certain assumptions (see text), rather than directly obtained.

when the Im/PIL mixture coexists with the mesoporous particles. Hence, to discuss the implications of the found values, $D_{\mathrm{Im}}$ is estimated for the case of the hybrid samples, assuming the same $D_{\mathrm{Im}} / D_{\mathrm{NH}}$ ratio as in the case of the neat $\mathrm{Im} / \mathrm{PIL}$ liquid mixture. This assumption is supported by the very similar $D_{\mathrm{NH}} / D_{\text {cat }}$ ratios observed, which reflect the occurrence of equivalent dynamics in the neat liquid mixture and in the hybrid materials (we also show by vibrational spectroscopy that the local interactions in terms of H-bonds are in fact similar in these two cases; see below). Furthermore, the estimated $D_{\operatorname{Im}}$ values reported in Table 3 are in very good agreement with those that one would obtain by treating the diffusion values experimentally measured for the resonances of the $\mathrm{C}^{2} \mathrm{H}$ and $\mathrm{C}^{4,5} \mathrm{H}$ protons as a population average of 1 octylimidazolium and imidazole species. This analytical approach was also implemented in one of our previous studies. ${ }^{1}$

The diffusion NMR results indicate that imidazolium, and under our assumptions also imidazole, move faster as the pore filling increases. More precisely, the 50 and the $100 \%$ samples, and the 200 and $400 \%$ samples, show pairwise similar behaviors. A particularly interesting observation is that the proton sitting on the $-\mathrm{NH}$ group, i.e., the most acidic site of the octyl-imidazolium cation, displays self-diffusion values systematically higher than that of the cation itself, suggesting a transport mechanism that is not exclusively vehicular. Here, we hypothesize the contribution of a proton motion decoupled from molecular diffusion, based on the fast exchange regime of the materials investigated (one merged signal for all involved exchangeable protons) and the fact that $D_{\mathrm{NH}}$ is higher than both $D_{\text {cat }}$ and $D_{\mathrm{Im}}$. Indeed, if a symmetrical two-site proton exchange reaction between imidazole and imidazolium was the only exceptional phenomenon occurring during the time of the diffusion measurement, the relation $D_{\text {cat }}<D_{\mathrm{NH}}<D_{\mathrm{Im}}$ would have been observed instead. Such specific dynamical conditions have recently been studied and discussed by us (using NMR methods) for a prototypical PIL/water mixture. ${ }^{44}$ With the intention to estimate the dynamics of protons moving faster than the parent molecules $\left(D_{\mathrm{H}}\right),{ }^{1,19}$ we make use of the following equation that assumes the $-\mathrm{NH}$ resonance to also include contributions from imidazole $\left(D_{\mathrm{Im}}\right)$ and imidazolium $\left(D_{\text {cat }}\right)$

$$
D_{\mathrm{NH}}=\chi_{1} D_{\mathrm{H}}+\chi_{2} D_{\mathrm{Im}}+\chi_{3} D_{\text {cat }}
$$

To make sure that the equation above is correctly used and $D_{\mathrm{H}}$ not overestimated, different phenomenological situations have been analyzed (see the extended discussion in the Supporting Information, "Treatment of Diffusion Data", and Table S5). In the most realistic scenario, one proton per added imidazole is enabled to diffuse faster than the parent molecule, via established and directional hydrogen bonds. This leads to setting $\chi_{1}$ equal to $\chi_{2}$, which is the molar fraction of added imidazole $\left(\chi_{2}=0.15\right)$ and, consequently, $\chi_{3}$ equal to 0.70 , which is the fraction of chemically bound $\mathrm{H}$ species that move exclusively in the molecular form of the imidazolium cation.

Based on the described scenario and by using the equation above, we find that the exchangeable protons diffuse significantly faster than their parent molecules, in the hybrid materials investigated here with a self-diffusion coefficient about 3-4 times faster than the imidazolium cation (see the $D_{\mathrm{H}} / D_{\text {cat }}$ column in Table 3). One should note, however, that these are averaged values that do not distinguish between diffusing species inside and outside the microparticles. The values obtained are very similar to those found for the case of microparticles with $10 \mathrm{~nm}$ pores, ${ }^{1}$ and supports the idea raised by us also in that previous work that the coexistence with mesoporous hydrophobic microparticles can result in enhanced dynamics. The self-diffusion coefficients discussed above are also shown in a graphical manner in Figure 9. It is interesting that at the transition between 100 and $200 \%$ of pore filling a sharp, rather than smooth, decrease is observed, whereas the hybrid materials with 200 and $400 \%$ of pore filling do not differ significantly from each other. From this observation, it appears that the liquid phases outside and inside the mesopores have distinct dynamical properties, with a $D_{\text {cat }}$ clearly higher outside. At this stage, we do not have a conclusive explanation for this difference, but a plausible aspect to investigate further is the difference in self-structuration of the ionic liquid in the high-curvature and concave mesopores as opposed to the flat outer surface of the microparticles as well as in the space in between these particles; see the simplified schematic in Figure 10. This difference is likely to induce a locally different molecular ordering and/or different ionic densities. Computational approaches may help elucidating this point, although most of the simulation studies of ionic liquids close to interfaces have so far typically considered flat interfacial surfaces, while the case of nanocavities remains much less explored. ${ }^{45,46}$ 


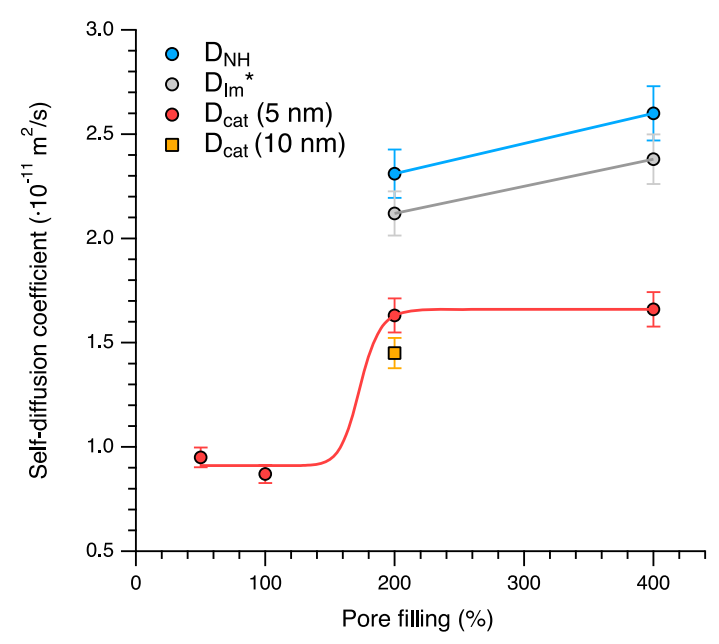

Figure 9. Self-diffusion coefficients experimentally measured $\left(D_{\text {cat }}\right.$ and $\left.D_{\mathrm{NH}}\right)$ or estimated $\left(D_{\mathrm{Im}}\right)$ shown as a function of pore filling. The asterisk represents the value estimated under certain assumptions (see main text) rather than directly obtained.

Molar Conductivities. To get a deeper insight into the transport mechanism of the ions in the investigated hybrid materials, we have estimated the molar conductivity, $\Lambda$, in the following two ways; from the conductivity data obtained by impedance spectroscopy, $\Lambda_{\text {imp }}$ (taking into account the volume fraction of silica and the ionic density), and from the selfdiffusion values, $\Lambda_{\mathrm{NMR}}$, using the Nernst-Einstein equation where $\Lambda_{\mathrm{NMR}}=2 D_{\text {cat }} \cdot F^{2} /(R T)$ and $F^{2} /(R T)$ at $30^{\circ} \mathrm{C}$ is equal to $3.6934 \times 10^{6} \mathrm{sS} / \mathrm{mol}$. In the latter, we use the previous result that $D_{\text {cation }} \simeq D_{\text {anion }}$ for the octylimidazolium-TFSI ionic liquid. ${ }^{47,48} \Lambda_{\text {imp }}$ and $\Lambda_{\mathrm{NMR}}$ are hence quantities estimated from two independent measurements. Moreover, while $\Lambda_{\text {imp }}$ represents a real absolute quantity, $\Lambda_{\mathrm{NMR}}$ is the maximum molar conductivity that one can expect assuming that all ions diffuse as dissociated species. In practice, however, ionic liquids usually have $\Lambda_{\mathrm{imp}} / \Lambda_{\mathrm{NMR}}$ ratios smaller than unity, which is also the reason why the ionicity of many ionic liquids falls below the ideal line of a Walden plot. Using the conductivity and diffusion data obtained for the Im/PIL mixture and the samples with 400,200 , and $100 \%$ of pore filling, we find values of $\Lambda_{\text {imp }} / \Lambda_{\mathrm{NMR}}$ equal to $0.79,0.22,0.17$, and 0.08 , respectively; see also Table S6 and the "Discussion of the Molar Conductivity" section in the Supporting Information. A value around 0.5 for the ionicity of ionic liquids (i.e., for the $\Lambda_{\text {imp }} /$ $\Lambda_{\mathrm{NMR}}$ ratio) is rather common, for instance, it is the value previously found for the pure ionic liquid 1-octylimidazolium TFSI by both Tokuda et al. ${ }^{47}$ and Martinelli et al. ${ }^{48}$ The decrease of this ratio upon confinement can be explained by an increasing fraction of paired ionic species inside the nanopores, the presence of less mobile species not contributing to $\Lambda_{\text {imp }}$ (i.e., the number of mobile ions may be smaller than expected from the nominal volume fraction) or a bad connectivity between liquid domains. In view of the structural results exposed below, the liquid layer self-assembled at the pore walls may possibly have a negative impact on $\Lambda_{\text {imp }}$, but cannot alone explain the extremely low $\Lambda_{\text {imp }} / \Lambda_{\mathrm{NMR}}$ values. We will come back to this point below.

Local Structure and Interactions. The most intriguing question is how the bulky molecules constituting the PIL, i.e., the TFSI anion and the octyl-imidazolium cation, organize inside pores only a few nanometers large and how this may relate to the observed transport properties. With the purpose of getting insight into the local structure within the pores, we have performed X-ray scattering experiments at room temperature for all samples under investigation. Focus has been on the $q$ range covered by wide-angle scattering, in practice the range $0.1-2.2 \AA^{-1}$ where long-chain ionic liquids typically show their characteristic features arising from nanostructuration. ${ }^{25,48}$ Figure 11 shows the change of the scattering pattern with

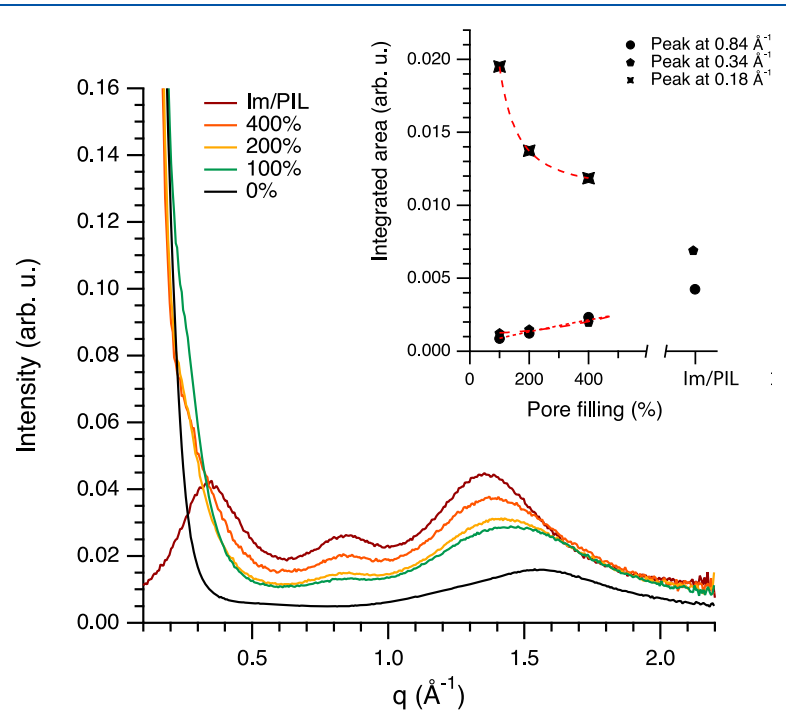

Figure 11. Small-angle X-ray scattering intensity collected for the bare silica particles (black trace), different samples with varying pore filling (orange, yellow, and green), and the neat Im/PIL mixture (dark red). The inset plot shows the integrated areas under selected fit peaks as a function of composition.

composition. The bulk Im/PIL liquid, i.e., the imidazole/ $\mathrm{HC}_{8}$ ImTFSI mixture, shows the three characteristic peaks to be centered at $1.35,0.84$, and $0.34 \AA^{-1}$, which have already been extensively presented and discussed for a number of protic and aprotic ionic liquids. ${ }^{25,49-51}$ Briefly, the peak at 0.84 $\AA^{-1}$ arises from all ion-ion alternations, while the peak at 0.34 $\AA^{-1}$ arises from the segregation of alkyl chains and corresponds to scattering domains about $1.8 \mathrm{~nm}$ in size. ${ }^{25,48}$ With respect to peak intensity and peak width, this feature is equivalent to the

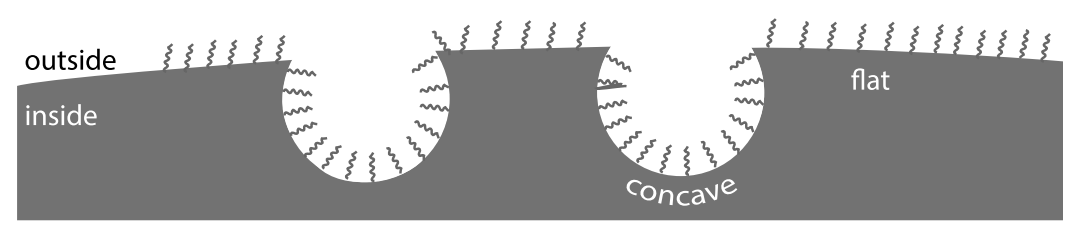

Figure 10. Schematic of the hydrophobized silica (with TOS groups anchored to its surface), emphasizing the different surface curvatures associated with the external (almost flat) and internal (concave) surfaces. 
case of the pure ionic liquid $\mathrm{HC}_{8} \mathrm{ImTFSI},{ }^{25}$ indicating that the addition of imidazole at a molar fraction of 0.15 does not disrupt the native local structure of the ionic liquid. The bare silica particles contribute at about $1.6 \AA^{-1}$ with a broader feature and with a strongly increasing intensity in the lowest $q$ range (below $0.3 \AA^{-1}$; see the black trace in Figure 11).

For the intermediate cases, i.e., the particles filled with the liquid phase (samples with filling factors of 400, 200, and $100 \%$ ), an additional feature below $0.4 \AA^{-1}$ is observed, which is better visualized in Figure S1A. This feature has been taken into account in the model used to fit the experimental data, which also included a linear background and individual contributions from silica and the liquid mixture, weighted to obtain the best fit results. Figure S1B demonstrates the good quality of the fits for these intermediate samples and that the additional feature was centered at $\sim 0.18 \AA^{-1}$ for all samples. Its intensity (i.e., the integrated area under the peak), however, increased for decreasing filling factors, concomitant with an intensity loss for the peak assigned to the chain-chain separation $\left(q \sim 0.34 \AA^{-1}\right)$ typical of the bulk liquid phase. These changes are visualized in the inset of Figure 11, which also shows that the intensity of the peak at $0.84 \AA^{-1}$ is less affected by composition. In fact, this can indicate that the local ion-ion distances are the same inside the mesopores as in the bulk liquid phase. Based on this information, it is realistic to assume a local structure inside the mesopores with one layer of ionic liquid self-assembled at the pore wall, and a higher density of charges (provided by the cation heads and the TFSI anions) toward the center of the pores in a domain whose size can match the observed distance of $\sim 3.5 \mathrm{~nm}$ (associated with the $\sim 0.18 \AA^{-1}$ feature). A sketch of this local structure is shown in Figure 12, while the hypothesized loose chain packing inside the pores is here rationalized based on the high curvature of the mesopores.

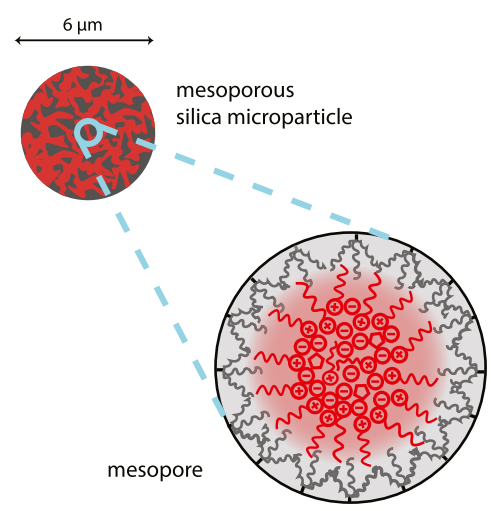

Figure 12. Illustration of the speculated local structure inside the mesopores, based on X-ray observations.

To complete the study and gain insight into the local interactions experienced by the nanoconfined liquid, vibrational spectroscopic methods were used. Raman spectroscopy, with focus on the vibrations of the TFSI anion sensitive to interactions, revealed that the conformational population of TFSI as well as the TFSI-octylimidazolium association were unchanged inside the mesopores compared to the case of the bulk Im/PIL liquid mixture (Figure 13). This also leads to the conclusion that the TFSI anion does not interact significantly with the silica walls, which is in line with the local structure obtained from X-ray scattering.

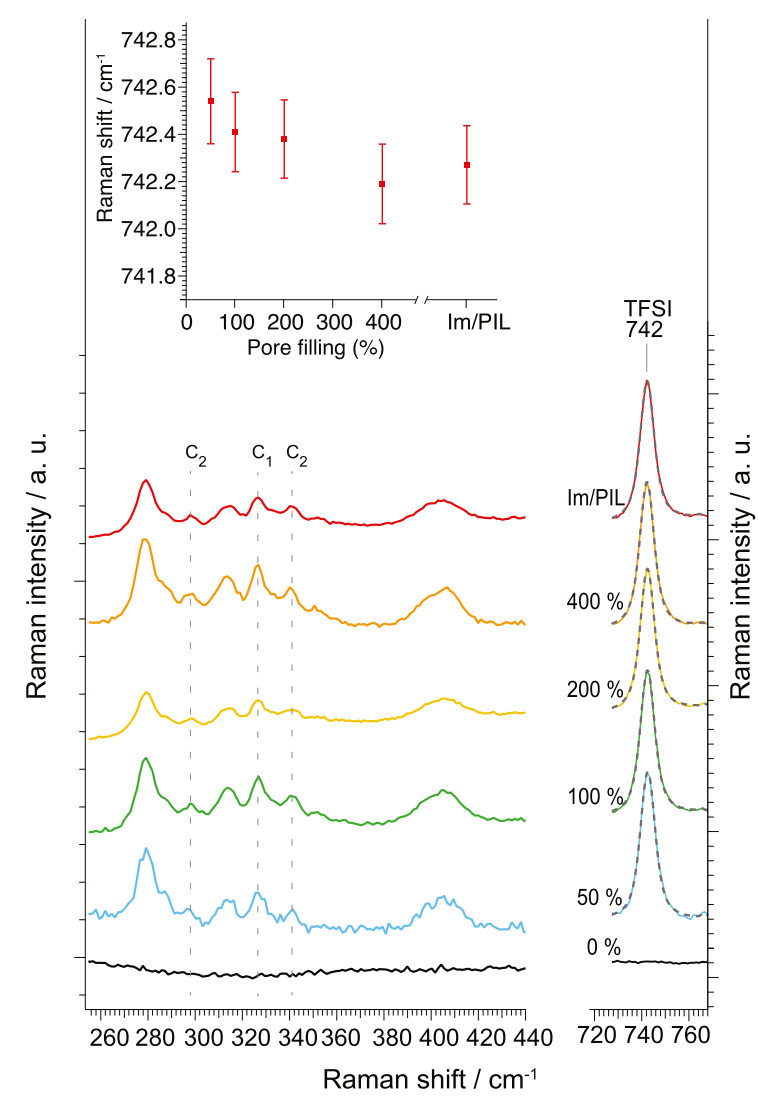

Figure 13. Selected regions of the Raman spectra collected for the $\mathrm{Im} / \mathrm{PIL}$ mixture and the mesoporous silica microparticles filled with this mixture at various degrees of pore filling. The dashed lines show the fitting by a Lorentzian function of the range $720-760 \mathrm{~cm}^{-1}$, where the expansion-contraction mode characteristic of the TFSI anion appears. The inset shows the frequency shift as a function of composition of this vibrational mode, which is sensitive to local intermolecular interactions. The error bars include the error of fitting and the precision range of the Raman spectrometer.

More precisely, the Raman shift range $240-450 \mathrm{~cm}^{-1}$ contains vibrational modes associated with the two conformational states of TFSI, ${ }^{52,53}$ i.e., the $\mathrm{C}_{1}$ (cisoid) and the $\mathrm{C}_{2}$ (transoid), that remain practically unchanged upon nanoconfinement (Figure 13). Furthermore, by peak fitting (see an example in Figure S6) we find that the expansion-contraction mode at $\sim 742 \mathrm{~cm}^{-1}$ does not broaden nor does it significantly shift in frequency with composition. The change in frequency with composition for this vibrational mode is shown in the inset of Figure 13 and indeed reveals to stay constant within the instrumental precision limits $\left( \pm 0.15 \mathrm{~cm}^{-1}\right)$. The increase in frequency upon lower degrees of filling is also much smaller than the blueshift of about $2 \mathrm{~cm}^{-1}$ observed for ionogels in correlation to strongly slowed down molecular dynamics. ${ }^{52}$ This is in line with a previous study by Garaga et al., ${ }^{2}$ which showed that due to what we called a flipped-ion effect in proximity to a hydrophobized silica wall (with the alkyl chain of the imidazolium cation facing the silica wall) the local interactions between the TFSI anion and the imidazolium head remain the same as in the pure PIL or the Im/PIL mixture. A different scenario, with a clear blueshift for the $\sim 742 \mathrm{~cm}^{-1}$ mode, has instead been observed in the case of untreated silica that offers $-\mathrm{SiO}^{-}$and $-\mathrm{SiOH}$ sites of interactions. ${ }^{52}$ In other words, the achieved spectroscopic information does not suggest the formation of ion pairs in the hybrid materials 
that could explain the much lower ionic conductivity compared to the Im/PIL mixture. As a consequence, we believe that discontinuities and bad connectivity in the long range and in between the particles are the main reason for the low $\Lambda_{\text {imp }}$ / $\Lambda_{\mathrm{NMR}}$ values measured and discussed above.

As a complement to Raman measurements, infrared spectroscopy was used to study the interaction sensitive vibrations of the imidazolium cation. Figures S7 and S8 reveal no significant changes for the $\mathrm{N}-\mathrm{H}$ stretching mode at about $3200 \mathrm{~cm}^{-1}$, confirming the results from Raman spectroscopy that the intermolecular interactions are practically the same throughout the different samples.

\section{CONCLUSIONS}

This study presents a multiple-technique characterization of potentially proton conductive organic-inorganic functional hybrid materials based on the imidazole $/ \mathrm{HC}_{8}$ ImTFSI mixture and hydrophobized mesoporous silica particles, with focus on the proton transport mechanism and the correlated local structure within the pores. We find that the ionic conductivity on a macroscopic scale is dominated by the vehicular mechanism, describable by the VFT equation, and that, as expected, it decreases rapidly as the silica content increases. For the materials with 200 and $400 \%$ of pore filling, the PFG NMR results show the occurrence of a proton motion decoupled from the imidazolium cation; however, for the samples with filling factors equal to 50 and $100 \%$, this phenomenon could not be verified due to the issues of line broadening. More precisely, for the higher filling factors, i.e., when the Im/PIL mixture is located also in between the particles, both protonic and ionic mobilities are enhanced. Since an average behavior has been measured, the question whether a decoupled proton motion also occurs inside the pores remains open.

In line with previous studies on the effect of hydrophobization of the silica pore walls, ${ }^{1}$ both vibrational spectroscopy and X-ray scattering show that the interactions at the molecular level are practically the same in the bulk liquid and in the hybrid materials. Hence, the flipped-ion effect involving the orientation of the nonpolar alkyl chain toward the hydrophobic silica pore wall is assumed to occur also in the smaller, about $5 \mathrm{~nm}$ wide, hydrophobized silica pores, similarly to the case of the $10 \mathrm{~nm}$ pores previously studied. ${ }^{1}$ As already shown before, ${ }^{1}$ in such structuration, the weak cation-silica interactions favor unrestricted diffusion, which is otherwise hindered under nanoconfinement and strong surface interactions. As a consequence, the low $\Lambda_{\text {imp }} / \Lambda_{\mathrm{NMR}}$ values recorded for the samples with a low pore filling reflect primarily the problem of long-range discontinuity and tortuous paths due to the presence of the microparticles. To conclude, in the scope of developing PIL-based proton conductive hybrid materials, this study confirms the beneficial effect of adding hydrophobized mesoporous silica on the protonic and ionic dynamics of an Im/PIL mixture. The hybrid materials presented in this work, in the regime of suspensions, could thus be functional in fuel cells operating at intermediate temperatures and anhydrous conditions; yet, the material concept may also be interesting in the context of Li-ion and Na-ion batteries.

\section{ASSOCIATED CONTENT}

\section{Supporting Information}

The Supporting Information is available free of charge at https://pubs.acs.org/doi/10.1021/acs.jpcc.0c08627.

Additional data on sample composition, centrifugation steps, complementary results from 2D Raman mapping measurements, discussion of the self-diffusion equation selected to analyze PFG NNR results, summary of relevant parameters used to estimate the molar conductivities, and examples of fit procedures applied to Raman and infrared spectra as well as to X-ray patterns (PDF)

\section{AUTHOR INFORMATION}

\section{Corresponding Author}

Anna Martinelli - Department of Chemistry and Chemical Engineering, Chalmers University of Technology, 41296 Gothenburg, Sweden; 10 orcid.org/0000-0001-9885-5901; Phone: +46 (0)31 772 3002; Email: anna.martinelli@ chalmers.se

\section{Authors}

Szilvia Vavra - Department of Chemistry and Chemical Engineering, Chalmers University of Technology, 41296 Gothenburg, Sweden; (1) orcid.org/0000-0003-0012-0507

Khalid Elamin - Department of Chemistry and Chemical Engineering, Chalmers University of Technology, 41296 Gothenburg, Sweden; Department of Chemistry and Molecular Biology, University of Gothenburg, 40530 Gothenburg, Sweden

Lars Evenäs - Department of Chemistry and Chemical Engineering, Chalmers University of Technology, 41296 Gothenburg, Sweden; 이이.0rg/0000-0002-6580-0610

Complete contact information is available at:

https://pubs.acs.org/10.1021/acs.jpcc.0c08627

\section{Notes}

The authors declare no competing financial interest.

\section{ACKNOWLEDGMENTS}

The authors acknowledge the financial support from the Swedish Foundation for Strategic Research (SSF, FFL-6 Program, Grant FFL-15 0092) and from the Knut \& Alice Wallenberg Foundation (Wallenberg Academy Fellows Program, Grant 2016-0220). The NMR measurements were carried out at the Swedish NMR Centre in Gothenburg, Sweden.

\section{REFERENCES}

(1) Garaga, M. N.; Dracopoulos, V.; Werner-Zwanziger, U.; Zwanziger, J. W.; Maréchal, M.; Persson, M.; Nordstierna, L.; Martinelli, A. A long-chain protic ionic liquid inside silica nanopores: Enhanced proton mobility due to efficient self-assembly and decoupled proton transport. Nanoscale 2018, 10, 12337-12348.

(2) Garaga, M. N.; Aguilera, L.; Yaghini, N.; Matic, A.; Persson, M.; Martinelli, A. Achieving enhanced ionic mobility in nanoporous silica by controlled surface interactions. Phys. Chem. Chem. Phys. 2017, 19, $5727-5736$

(3) Garaga, M. N.; Persson, M.; Yaghini, N.; Martinelli, A. Local coordination and dynamics of a protic ammonium based ionic liquid immobilized in nano-porous silica micro-particles probed by Raman and NMR spectroscopy. Soft Matter 2016, 12, 2583-2592. 
(4) Zhang, S.; Zhang, J.; Zhang, Y.; Deng, Y. Nanoconfined Ionic Liquids. Chem. Rev. 2017, 117, 6755-6833.

(5) Ingenmey, J.; Gehrke, S.; Kirchner, B. How to Harvest Grotthuss Diffusion in Protic Ionic Liquid Electrolyte Systems. ChemSusChem 2018, 11, 1900-1910.

(6) Hasani, M.; Varela, L. M.; Martinelli, A. Short-Range Order and Transport Properties in Mixtures of the Protic Ionic Liquid [C2HIm][TFSI] with Water or Imidazole. J. Phys. Chem. B 2020, 124, 1767-1777.

(7) Amarasekara, A. S. Acidic Ionic Liquids. Chem. Rev. 2016, 116, 6133-6183.

(8) Singh, M. P.; Singh, R. K.; Chandra, S. Ionic liquids confined in porous matrices: Physicochemical properties and applications. Prog. Mater. Sci. 2014, 64, 73-120.

(9) Kreuer, K. D.; Paddison, S. J.; Spohr, E.; Schuster, M. Transport in proton conductors for fuel-cell applications: Simulations, elementary reactions, and phenomenology. Chem. Rev. 2004, 104, 4637-4678.

(10) Le Bideau, J.; Viau, L.; Vioux, A. Ionogels, ionic liquid based hybrid materials. Chem. Soc. Rev. 2011, 40, 907-925.

(11) Duan, C.; Majumdar, A. Anomalous ion transport in 2-nm hydrophilic nanochannels. Nat. Nanotechnol. 2010, 5, 848-852.

(12) Li, X.; Zhang, H.; Yu, H.; Xia, J.; Zhu, Y. B.; Wu, H. A.; Hou, J.; Lu, J.; Ou, R.; Easton, C. D.; et al. Unidirectional and Selective Proton Transport in Artificial Heterostructured Nanochannels with Nano-toSubnano Confined Water Clusters. Adv. Mater. 2020, 32, No. 2001777

(13) Ma, X.; Li, C.; Martinson, A. B.; Voth, G. A. Water-Assisted Proton Transport in Confined Nanochannels. J. Phys. Chem. C 2020, 124, 16186-16201.

(14) Verma, Y. L.; Singh, M. P.; Kumar, S.; Dhar, R.; Singh, R. K. Behaviour of ionic liquid adsorbed on the surface of nano silica particles and in confined system of silica matrices. Surf. Sci. 2020, 701, No. 121701.

(15) Tomita, K.; Mizukami, M.; Nakano, S.; Ohta, N.; Yagi, N.; Kurihara, K. X-Ray diffraction and resonance shear measurement of nano-confined ionic liquids. Phys. Chem. Chem. Phys. 2018, 20, 13714-13721.

(16) Rouha, M.; Cummings, P. T. Thickness-dependent structural arrangement in nano-confined imidazolium-based ionic liquid films. Phys. Chem. Chem. Phys. 2015, 17, 4152-4159.

(17) Perkin, S.; Albrecht, T.; Klein, J. Layering and shear properties of an ionic liquid, 1-ethyl-3- methylimidazolium ethylsulfate, confined to nano-films between mica surfaces. Phys. Chem. Chem. Phys. 2010, $12,1243-1247$.

(18) Sippel, P.; Lunkenheimer, P.; Krohns, S.; Thoms, E.; Loidl, A. Importance of liquid fragility for energy applications of ionic liquids. Sci. Rep. 2015, 5, No. 13922.

(19) Yaghini, N.; Gómez-González, V.; Varela, L. M.; Martinelli, A. Structural origin of proton mobility in a protic ionic liquid/imidazole mixture: Insights from computational and experimental results. Phys. Chem. Chem. Phys. 2016, 18, 23195-23206.

(20) Iacob, C.; Sangoro, J. R.; Papadopoulos, P.; Schubert, T.; Naumov, S.; Valiullin, R.; Kärger, J.; Kremer, F. Charge transport and diffusion of ionic liquids in nanoporous silica membranes. Phys. Chem. Chem. Phys. 2010, 12, 13798-13803.

(21) Iacob, C.; Sangoro, J. R.; Kipnusu, W. K.; Valiullin, R.; Kärger, J.; Kremer, F. Enhanced charge transport in nano-confined ionic liquids. Soft Matter 2012, 8, 289-293.

(22) Verma, Y. L.; Singh, R. K. Conformational States of Ionic Liquid 1-Ethyl-3-methylimidazolium Bis(trifluoromethylsulfonyl)imide in Bulk and Confined Silica Nanopores Probed by Crystallization Kinetics Study. J. Phys. Chem. C 2015, 119, 2438124392.

(23) Elola, M. D.; Rodriguez, J. Ionic Mobility within Functionalized Silica Nanopores. J. Phys. Chem. C 2019, 123, 3622-3633.

(24) Hayes, R.; Warr, G. G.; Atkin, R. Structure and Nanostructure in Ionic Liquids. Chem. Rev. 2015, 115, 6357-6426.
(25) Abdurrokhman, I.; Elamin, K.; Danyliv, O.; Hasani, M.; Swenson, J.; Martinelli, A. Protic Ionic Liquids Based on the AlkylImidazolium Cation: Effect of the Alkyl Chain Length on Structure and Dynamics. J. Phys. Chem. B 2019, 123, 4044-4054.

(26) Thommes, M.; Kaneko, K.; Neimark, A. V.; Olivier, J. P.; Rodriguez-Reinoso, F.; Rouquerol, J.; Sing, K. S. Physisorption of gases, with special reference to the evaluation of surface area and pore size distribution (IUPAC Technical Report). Pure Appl. Chem. 2015, 87, 1051-1069.

(27) Staudt, P. B.; Kechinski, C. P.; Tessaro, I. C.; Marczak, L. D.; De, R.; Cardozo, N. S. A new method for predicting sorption isotherms at different temperatures using the BET model. J. Food Eng. 2013, 114, 139-145.

(28) Anderson, M. S. Nonplasmonic surface enhanced Raman spectroscopy using silica microspheres. Appl. Phys. Lett. 2010, 97, No. 131116.

(29) Merlen, A.; Sangar, A.; Torchio, P.; Kallepalli, L. N.; Grojo, D.; Utéza, O.; Delaporte, P. Multi-wavelength enhancement of silicon Raman scattering by nanoscale laser surface ablation. Appl. Surf. Sci. 2013, 284, 545-548.

(30) Tsang, J. C.; Kirtley, R.; Bradley, J. A. Surface-Enhanced Raman Spectroscopy and Surface Plasmons. Phys. Rev. Lett. 1979, 43, No. 772.

(31) Ausman, L. K.; Schatz, G. C. Whispering-gallery mode resonators: Surface enhanced Raman scattering without plasmons. J. Chem. Phys. 2008, 129, No. 054704.

(32) Mitra, S.; Cerclier, C.; Berrod, Q.; Ferdeghini, F.; de OliveiraSilva, R.; Judeinstein, P.; le Bideau, J.; Zanotti, J. M. Ionic liquids confined in silica ionogels: Structural, thermal, and dynamical behaviors. Entropy 2017, 19, No. 140.

(33) Göbel, R.; Hesemann, P.; Weber, J.; Eleonore, M.; Alwin, F.; Taubert, A.; Beuermanna, S. Surprisingly high, bulk liquid-like mobility of silica-confined ionic liquids. Phys. Chem. Chem. Phys. 2009, 11, 3653-3662.

(34) Lin, J.; Korte, C. PBI-type Polymers and Acidic Proton Conducting Ionic Liquids - Conductivity and Molecular Interactions. Fuel Cells 2020, 20, 461-468.

(35) Shin, J. H.; Henderson, W. A.; Passerini, S. Ionic liquids to the rescue? Overcoming the ionic conductivity limitations of polymer electrolytes. Electrochem. Commun. 2003, 5, 1016-1020.

(36) Kitazawa, Y.; Iwata, K.; Kido, R.; Imaizumi, S.; Tsuzuki, S.; Shinoda, W.; Ueno, K.; Mandai, T.; Kokubo, H.; Dokko, K.; et al. Polymer Electrolytes Containing Solvate Ionic Liquids: A New Approach to Achieve High Ionic Conductivity, Thermal Stability, and a Wide Potential Window. Chem. Mater. 2018, 30, 252-261.

(37) Elamin, K.; Shojaatalhosseini, M.; Danyliv, O.; Martinelli, A.; Swenson, J. Conduction mechanism in polymeric membranes based on PEO or PVdF-HFP and containing a piperidinium ionic liquid. Electrochim. Acta 2019, 299, 979-986.

(38) Kösters, J.; Schönhoff, M.; Stolwijk, N. A. Mass and charge transport in the polymer-ionic-liquid system PEO-EMImI: From ionic-liquid-in-polymer to polymer-in-ionic-liquid electrolytes. J. Phys. Chem. B 2015, 119, 5693-5700.

(39) Meng, X.; Wang, H. N.; Song, S. Y.; Zhang, H. J. Protonconducting crystalline porous materials. Chem. Soc. Rev. 2017, 46, $464-480$.

(40) Sun, X.; Simonsen, S. C.; Norby, T.; Chatzitakis, A. Composite membranes for high temperature PEM fuel cells and electrolysers: A critical review. Membranes 2019, 9, No. 83.

(41) Wojnarowska, Z.; Feng, H.; Diaz, M.; Ortiz, A.; Ortiz, I.; Knapik-Kowalczuk, J.; Vilas, M.; Verdía, P.; Tojo, E.; Saito, T.; et al. Revealing the Charge Transport Mechanism in Polymerized Ionic Liquids: Insight from High Pressure Conductivity Studies. Chem. Mater. 2017, 29, 8082-8092.

(42) Robinson, N.; D'Agostino, C. NMR Investigation into the Influence of Surface Interactions on Liquid Diffusion in a Mesoporous Catalyst Support. Top. Catal. 2019, 319-327.

(43) Barrie, P. J. Characterization of porous media using NMR methods. Annu. Rep. NMR Spectrosc. 2000, 41, 265-278. 
(44) Hasani, M.; Nordstierna, L.; Martinelli, A. Molecular dynamics involving proton exchange of a protic ionic liquid-water mixture studied by NMR spectroscopy. Phys. Chem. Chem. Phys. 2019, 21, 22014-22021.

(45) Ori, G.; Villemot, F.; Viau, L.; Vioux, A.; Coasne, B. Ionic liquid confined in silica nanopores: Molecular dynamics in the isobaricisothermal ensemble. Mol. Phys. 2014, 112, 1350-1361.

(46) Singh, R.; Monk, J.; Hung, F. R. Heterogeneity in the dynamics of the ionic liquid [BMIM+] [PF6-] confined in a slit nanopore. J. Phys. Chem. C 2011, 115, 16544-16554.

(47) Tokuda, H.; Hayamizu, K.; Ishii, K.; Susan, M. A. B. H.; Watanabe, M. Physicochemical properties and structures of room temperature ionic liquids. 2. variation of alkyl chain length in imidazolium cation. J. Phys. Chem. B 2005, 109, 6103-6110.

(48) Martinelli, A.; Maréchal, M.; Östlund, Å.; Cambedouzou, J. Insights into the interplay between molecular structure and diffusional motion in 1-alkyl-3-methylimidazolium ionic liquids: A combined PFG NMR and X-ray scattering study. Phys. Chem. Chem. Phys. 2013, $15,5510-5517$.

(49) Triolo, A.; Russina, O.; Bleif, H. J.; Di Cola, E. Nanoscale segregation in room temperature ionic liquids. J. Phys. Chem. B 2007, 111, 4641-4644.

(50) Russina, O.; Triolo, A.; Gontrani, L.; Caminiti, R. Mesoscopic structural heterogeneities in room-temperature ionic liquids. J. Phys. Chem. Lett. 2012, 3, 27-33.

(51) Pádua, A. A. H.; Costa Gomes, M. F.; Canongia Lopes, J. N. Molecular solutes in ionic liquids: A structural perspective. Acc. Chem. Res. 2007, 40, 1087-1096.

(52) Nayeri, M.; Aronson, M. T.; Bernin, D.; Chmelka, B. F.; Martinelli, A. Surface effects on the structure and mobility of the ionic liquid C 6C1ImTFSI in silica gels. Soft Matter 2014, 10, 5618-5627. (53) Martinelli, A.; Matic, A.; Johansson, P.; Jacobsson, P.; Börjesson, L.; Fernicola, A.; Panero, S.; Scrosati, B.; Ohno, H. Conformational evolution of TFSI- in protic and aprotic ionic liquids. J. Raman Spectrosc. 2011, 42, 522-528. 